\title{
A face serum containing bakuchiol, palmitoyl tripeptide-38, hy- drolyzed hyaluronic acid and a polyherbal and vitamin blend improves skin quality in human volunteers and protects skin structure in vitro
}

\author{
Brett J. West ${ }^{1, *}$, Ife Alabi ${ }^{2}$ and Shixin Deng ${ }^{1}$ \\ 1 Research and Development, NewAge, Inc., American Fork, Utah, USA; brett_west@newage.com (B. West), \\ shixin_deng@newage.com (S. Deng) \\ 2 Research and Development, NewAge, Inc., Bray, Republic of Ireland; ife_alabi@newage.com \\ * Correspondence: brett_west@newage.com; Tel.: 1 (801) 234-1000
}

\begin{abstract}
A face serum composed of a combination of biologically active compounds was evaluated for safety and efficacy in vitro, in a repeat insult patch test and in a human clinical efficacy trial. The serum inhibited tyrosinase activity modestly, decreased collagenase activity and exhibited notable free radical scavenging activity in vitro. It is gentle to the skin, as the serum did not irritate the skin or produce symptoms of allergic contact dermatitis in the 55 healthy adults that participated in the repeat insult patch test. In the efficacy trial, daily application of the face serum for 30 days significantly increased skin hydration, with all 35 volunteers experiencing improvement. Substantial improvements in skin elasticity, roughness (fine lines and wrinkles), and brightness also occurred during the trial. Dermatological examination also revealed a trend for reduced comedone count with use of the serum. Self-assessment responses revealed that all volunteers experienced improvements in multiple skin quality parameters and that participant perceptions are consistent with the results of the instrumental analyses. These findings indicated that the measured improvements in skin quality are not only statistically significant but are also clinically relevant as they were great enough for users of the face serum to feel and recognize.
\end{abstract}

Keywords: face serum; bakuchiol; palmitoyl tripeptide-38; hyaluronic acid; repeat insult patch test; tyrosinase; collagenase; antioxidant; clinical trial

\section{Introduction}

The skin is a major protective organ of the human body. It provides first-line protection against numerous potential threats in our environment. The skin also helps regulate the temperature of our bodies, is the site of vitamin D synthesis and can produce certain hormones [1]. The skin is an important means of social communication, and the status of skin health can have a significant psychosocial impact individually as well as affect social interactions [2-5]. Further, skin quality has become a major indicator of social worth in many modern societies [6]. As such, efforts to maintain skin health will benefit physical and mental health as well interpersonal relationships.

Many extrinsic and intrinsic factors can accelerate the appearance of aging skin. Excessive UV light exposure, environment, lifestyle habits and genetic predisposition all influence the quality of human skin. A decrease in skin radiance, development of uneven melanin distribution and appearance of rhytides and telangiectasia are examples of visible signs of underlying degradation of extracellular matrix during the aging process. Topical skin treatments may potentially slow these skin changes and, perhaps, even restore the skin to a previous state of quality. Among the substances that may promote skin health 
are plant-derived compounds. One notable example is bakuchiol, a meroterpene which occurs in Psoralea corylifolia. Bakuchiol exhibits antioxidant and anti-inflammatory activities as well as possesses retinol-like antiaging properties but without the associated adverse effects $[7,8]$.

Terminalia ferdinandiana (Kakadu plum) is a source of several antioxidant compounds including vitamin $\mathrm{C}$ which, when topically applied, protects against UV-induced photo aging by possibly increasing gene expression of the tissue inhibitor of matrix metalloproteinases $[9,10]$. Shea butter, which is the phytosterol and tocopherol-rich fat extracted from the nut of the Vitellaria paradoxa tree, has long been used in sub-Saharan Africa as a folk remedy for dermatological disorders and has also found wide acceptance as an emollient which helps maintain skin barrier integrity [11, 12]. Perhaps, Aloe vera (syn. Aloe barbadensis) is one of the best know natural products for skin health. Topical Aloe vera gel may aid burn and wound healing as well as act as an anti-inflammatory agent [13]. Allantoin, derived from several plant sources, regulates inflammation and induces both fibroblast proliferation and extracellular matrix synthesis [14]. Case reports indicated that topical application of grape seed oil reduced the appearance of scaring and dark circles under the eyes [15].

Peptides have also demonstrated anti-aging potential. Due to their role in cell signaling within the skin, peptides have been investigated in the past few decades for topical benefits. Specific peptides have been developed to cross the stratum corneum and influence extracellular matrix synthesis, pigmentation, immunity and inflammation [16]. Palmitoyl tripeptide-38 is one such lipopeptide which is reported to increase the production of dermal and epidermal collagen, fibronectin and hyaluronic acid [17].

Vitamin C (ascorbic acid) improves skin elasticity and reduces wrinkles by stimulating collagen synthesis. It inhibits tyrosinase, which is responsible for the formation of melanin. It also protects the skin from oxidative stress by donating electrons to reactive oxygen species (ROS) neutralizing their effect. However, ascorbic acid is an extremely unstable compound. Ascorbyl phosphate salts, such as sodium ascorbyl phosphate (SAP), are among one of the most stable ascorbic acid derivatives. This due to the introduction of the phosphate group that protects the enediol system of the molecule against oxidation. It is converted to free vitamin $\mathrm{C}$ by enzymes present in the skin. The liposomal form of SAP not only further enhances stability but also exhibits greater penetration through the epidermis, thereby exerting greater effect on skin cells [18].

Hyaluronic acid is a component of the skin and integral to skin function via its ability to retain water. As the skin ages, epidermal hyaluronic acid content dramatically declines. The disappearance of this water-binding molecule results in the characteristic age-associated decrease in skin hydration status [19]. Unsurprisingly, topical application of sodium hyaluronate and other forms of hyaluronic acid have been found to effectively improve skin hydration, resulting in improvements in various facets of skin quality [20].

The purpose of our investigation was to evaluate both the safety and efficacy of a face serum developed with the substances discussed above. The serum was formulated to provide a comprehensive anti-aging topical treatment which would mitigate the adverse effects of environmental and intrinsic factors on the extracellular matrix as well as reduce some of the visible effects of aging. Such a formulation could aid users in their efforts to maintain a youthful appearance and promote overall well-being.

\section{Materials and Methods}

\subsection{Test material}

A face serum (Lucim) was formulated and prepared for daily application to the face. The serum contained the following ingredients: water, glycerin, grape seed oil, niacinamide, propanediol, silica, shea butter, glycerol oleate citrate, cetyl alcohol, bakuchiol, sucrose stearate, sodium ascorbyl phosphate, tocopherol, hydrolyzed hyaluronic acid, sodium hyaluronate, Terminalia ferdinandiana (Kakadu plum) fruit extract, palmitoyl tripeptide-38, allantoin, xanthan gum, alcohol, caprylyl glycol, phytic acid, 1,2-hexandiol, 
coconut alkanes, coco-caprylate/caprate, hydroxypropyl cyclodextrin, sorbitol, sodium hydroxide, Aloe barbadensis leaf juice and glyceryl caprylate. The serum was prepared in accordance with internationally recognized good manufacturing practices. To ensure product safety, microbiological analyses were performed on samples of the serum following production and an antimicrobial effectiveness test was conducted in accordance with harmonized US and EU protocols.

\subsection{Repeat insult patch test}

Fifty-five volunteers (13 males and 42 females, age 18 to 79 years) were enrolled in a repeat insult patch test of the face serum. The inclusion criteria for the repeat insult patch test were healthy adult volunteers with no physical or skin conditions which would interfere with the ability to discern potential skin reactions. During the induction phase, 0.2 $\mathrm{mL}$ of the face serum was placed on cotton fabric patch and applied for 24 hours to the skin of the back, with semi-occlusive surgical tape, adjacent to the spinal and between the scapulae and the waist. For each volunteer, application occurred 3 times a week with 24hour rest periods between each application, except for a 48-hour rest period between the final weekly application and the first application of the following week. The procedure was followed for 3 weeks, resulting in 9 applications during the induction phase. Two weeks after completion of the induction phase, a patch containing the face serum was applied to a previously unexposed section of skin. The skin of each subject was evaluated during this challenge phase at 24 and 72 hours following the final application of the face serum for possible delayed reactions, including redness, itching, and irritation. The skin of the volunteers was scored for reactions according to the International Contact Dermatitis Research Group and under the supervision of a consulting dermatologist [21]. Reactions were scored on a scale of 0 (no reaction) to 4 (deep red erythema, with or without vesiculation or weeping).

\subsection{Tyrosinase inhibition assay}

In vitro tyrosinase inhibition was evaluated according to a previously described method, but with modifications [22]. Various concentrations of sample were dissolved in $180 \mu \mathrm{L}$ phosphate buffer (193 mM KH2PO4, $73.4 \mathrm{mM} \mathrm{Na2CO3,} \mathrm{pH} \mathrm{7.2)} \mathrm{with} \mathrm{0.5} \mathrm{mM} \mathrm{L-}$ dopa (Sigma-Aldrich, St. Louis, Missouri) and 1400 units tyrosinase (Sigma-Aldrich, St. Louis, Missouri) and incubated at room temperature. A 100\% activity blank (no sample) was also prepared. These mixtures were incubated at room temperature for 15 minutes. Afterwards, absorbance was read at $450 \mathrm{~nm}$ in a microplate reader. Replicate samples were evaluated at each concentration. Tyrosinase inhibition activity was calculated from difference between $100 \%$ activity (no sample) and sample absorbance, divided by the absorbance of the $100 \%$ activity alone.

\subsection{Collagenase inhibition assay}

Inhibition of collagenase activity was assessed using an assay kit obtained from Sigma-Aldrich. In this assay, samples were prepared at various concentrations with deionized water and filtered via $0.45 \mu \mathrm{M}$ syringe filter. The dissolved samples $(2 \mu \mathrm{L})$ or a solvent blank (initial enzyme activity) were combined with $10 \mu \mathrm{L}$ collagenase solution ( 0.35 units $/ \mathrm{mL}$ ) and $88 \mu \mathrm{L}$ buffer in a clear polystyrene microwells then incubated at room temperature for 10 minutes. Next, an equal volume $(100 \mu \mathrm{L})$ of assay buffer, containing the collagenase substrate N-(3-[2-Furyl]acryloyl)-Leu-Gly-Pro-Ala (FALGPA), was added to each well containing samples or blank. The absorbance of each reaction mixture was measured at $345 \mathrm{~nm}$ in kinetic mode (every minute for 15 minutes) while incubating at 37 ${ }^{\circ} \mathrm{C}$. The area under the curve (AUC) for the absorbance vs. time of each sample or blank was calculated. AUC values were used to calculate inhibition, which was the difference 
between values for the blank and each sample, divided by that of the blank alone. Blanks and samples were evaluated in triplicate.

\subsection{2,2-Diphenylpicrylhydrazyl (DPPH) radical scavenging assay}

The 2,2-Diphenylpicrylhydrazyl (DPPH) radical scavenging assay were performed according to a method reported previously, but with some modification [23]. In the DPPH test, samples of increasing concentrations were prepared with aqueous ethanol. Next, sample solutions were combined 1:1 (v/v) with $0.4 \mathrm{mM} \mathrm{DPPH}$ in ethanol. The absorbance of each sample and blank (solvent only) was read at $515 \mathrm{~nm}$ after incubation at $37^{\circ} \mathrm{C}$ for $1 \mathrm{hr}$. Percent radical scavenging activity was calculated by dividing the difference in blank absorbance and sample by the absorbance of the blank alone. In the in vitro assays, sample concentrations are reported on dry weight basis (water weight excluded).

\subsection{Efficacy clinical trial}

A one-month clinical trial involving 30 healthy volunteers was conducted with the face serum. All participants agreed to not use other topical products on the face 7 days prior to the start of the study as well as during remainder of the study period. Volunteers excluded from the trial were pregnant or lactating women, those who had cosmetic facial treatments within the previous 4 weeks, those undergoing immunosuppressive therapy, current use of topical medications, allergy or hypersensitivity to one of the face serum ingredients, or a medical history of atopy, keloids, or post-inflammatory hyperpigmentation. The demographics of those enrolled in the trial were age range of 35 to 55 years, 23 females of European ancestry, 2 females of both European and African ancestry, 4 females of Asian ancestry, and 1 male of Asian ancestry. The percentage of facial skin types among the participants were $15 \%$ dry, $22 \%$ normal, $63 \%$ combination and none with oily skin.

The face serum was applied to the face once daily for 4 weeks. Visual evaluation by a dermatologist was performance before the start of the trial (T0), and after 4 weeks (T2) of facial application of the face serum. Clinical scores were given to initial and final conditions of the facial skin (forehead, cheeks, cheekbone, noes and chin) of each volunteer regarding the number of comedones and inflammatory lesions.

Instrumental analysis of the skin was performed at T0, two weeks after daily use of the face serum (T1) and at T2. Skin elasticity was measured with the Cutometer dual MPA 580 (Courage+Khazaka, Cologne, Germany) using mode 1, with a probe (model CT580MP) aperture of $2 \mathrm{~mm}$ and $450 \mathrm{mbar}$ negative pressure (suction). Total skin recovery at the end of deformation cycles (Ua), immediate skin retraction (Ur), and final skin deformation (Uf), all measured in millimeters, were used to calculate skin elasticity $\mathrm{R}$ values. Biological elasticity (R2) is the ratio $\mathrm{Ua} / \mathrm{Uf}$, while relative elastic recovery (R7) is the ratio Ur/Uf [24].

Skin hydration was measured with a Corneometer ${ }^{\circledR}$ CM 825 probe (Courage+Khazaka). This is one of the most well-documented non-invasive methods for reproducibly and accurately measuring the moisture level of the stratum corneum [25]. This method is based on capacitance measurements of the skin, in which the dielectric constant will change with water content. The probe is applied to the surface of the skin at a constant pressure of the measurement probe on the skin surface is 3.5 newtons, covers an area of $49 \mathrm{~mm} 2$, and measures water content up to a depth of $100 \mu \mathrm{m}$. Skin hydration measurements were conducted in a clinic room maintained at $22^{\circ} \mathrm{C} \pm 2{ }^{\circ} \mathrm{C}$ and $50 \%$ relative humidity. Skin hydration measurements were reported in corneometer units (CU).

Facial skin roughness (lateral canthal lines or wrinkles) was measured with DermaTOP Blue (Eotech SA, Marcoussis, France), a blue LED device for non-contact 3D analysis of skin roughness via fringe projection and optical triangulation. This allows for the measurement of skin wrinkles without the need to product silicone skin replicates. The roughness ( $R$ ) parameters $\mathrm{Rz}$ and $\mathrm{Ra}$, both measured in $\mathrm{mm}$, were evaluated. $\mathrm{Rz}$ is defined 
as the average wrinkle height/depth. Ra is the average wrinkle roughness, calculated from wrinkle width and height [26].

Skin brightness (specular brightness rate) was measured with a FrameScan ${ }^{\circledR}$ (ORION Concept, Tours, France). Changes in reflected light, which is relative to skin brightness, were measured with this system and used to calculate specular brightness rates. All instrument measurements performed under the same conditions as those use for skin hydration measurements.

At the end of the trial participants were asked to complete a self-assessment evaluation wherein they were instructed to look in a mirror and complete a questionnaire on noticeable differences in the appearance of their facial skin face and on their perception of efficacy and pleasantness face serum.

Summary statistics, such as mean and standard deviation, were calculated for each of the measurements. Data were evaluated for normality with the Shapiro-Wilk test followed by the paired Student's t-test for differences among measurements taken at T0, T1 and T2. Where data was not normally distributed, the Wilcoxon signed-rank test was used to make comparisons. The percent change in each measurement for individual participants was also determined and used to calculate average \% change. Both the efficacy trial and the repeat insult patch test were conducted in accordance with the World Medical Association's Declaration of Helsinki, and written informed consent was obtained from all participants.

\section{Results}

3.1 Repeat insult patch test

All 55 subjects completed the repeat insult patch test. There were no reactions observed during the induction phase. Further, there were no clinically significant responses during the challenge phase. As such, the face serum is not associated with skin irritation or allergic contact dermatitis.

\subsection{Tyrosinase inhibition assay}

In vitro tests of the face serum reveal at least a few potentially effective mechanisms of action. Anti-tyrosinase activity was found to be concentration-dependent but very modest. Tyrosinase was inhibited as low as $25 \mu \mathrm{g} / \mathrm{mL}$. However, no more than $20 \%$ inhibition was achieved at a concentration of $1 \mathrm{mg} / \mathrm{mL}$.

\subsection{Collagenase inhibition assay}

The face serum was more active against collagenase, demonstrating linear and concentration-dependent inhibition (Figure 1). Inhibition of collagenase was still evident at 3 $\mu \mathrm{g} / \mathrm{mL}$, and half-maximal inhibition (IC50) was achieved by $300 \mu \mathrm{g} / \mathrm{mL}$.

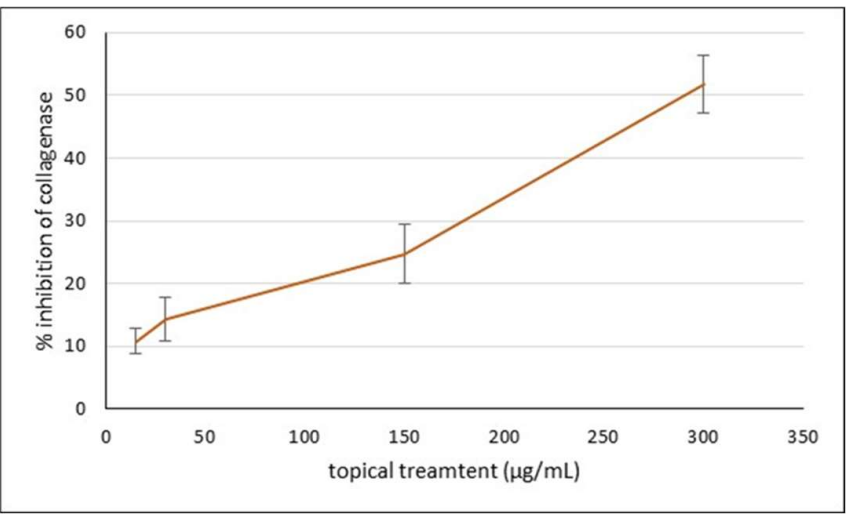


Figure 1. Inhibition of collagenase by face serum.

\subsection{2,2-Diphenylpicrylhydrazyl (DPPH) radical scavenging assay}

The face serum was much more active in the DPPH radical scavenging assay (Figure $2)$. It reduced free radicals in a concentration dependent manner, where the average ( \pm standard deviation) activity was $92.72 \pm 0.0006 \%$ at $150 \mu \mathrm{g} / \mathrm{mL}$. Even at a concentration as low as $10 \mu \mathrm{g} / \mathrm{mL}$, the actives in the face serum were able to neutralize approximately $20 \%$ of the free radicals in this assay.

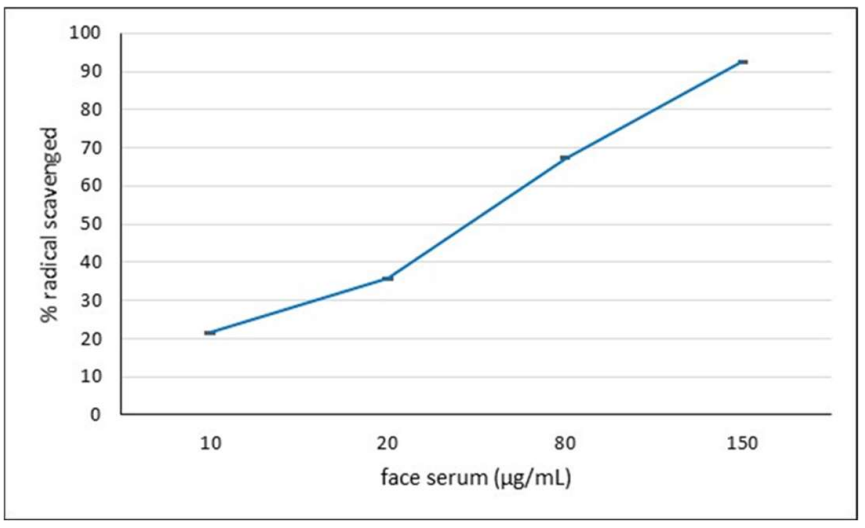

Figure 2. DPPH radical scavenging test of face serum.

\subsection{Efficacy clinical trial}

The average ( \pm standard deviation) of parameters measured during the trial are presented in Table 1. Over the course of the efficacy trial, all participants experienced increases in skin hydration. After 2 weeks of face serum use, average skin moisture among the volunteers increased $9.28 \%$, from (mean \pm standard deviation) $41.853 \pm 12.391$ to 45.740 $\pm 11.356 \mathrm{CU}, \mathrm{p}<0.001$. By the end of the trial, average skin moisture increased $23.82 \%$ from baseline (up to $50.520 \pm 11.823, p<0.001$ ). The averages of the percent change experienced by individual participants during the first and second halves of the trial were approximately equal ( $11.59 \%$ and $23.82 \%$, respectively), demonstrating a consistent response to the moisturizing properties of the face serum.

Skin elasticity increased in $97 \%$ of participants after 30 days. Mean biological elasticity (R2) increased 10\% after 2 weeks of face serum use (from $0.556 \pm 0.060$ to $0.609 \pm 0.062$, $\mathrm{p}<0.001)$. Average R2 increased further to $0.641 \pm 0.054(\mathrm{p}<0.001)$ after 30 days, where the average of individual percent increase was $15 \%$ above baseline (T0). The mean elastic recovery ability of the skin (R7) improved by approximately $8 \%$ within the first two weeks of the trial (from $0.382 \pm 0.055$ to $0.414 \pm 0.067, \mathrm{p}<0.01$ ). As with R2 values, average R7 continued to improve until the end of the trial where it increased by about $13 \%$ (up to $0.430 \pm 0.047, \mathrm{p}<0.01$ ). Improvements in $\mathrm{R} 2$ and $\mathrm{R} 7$ demonstrate improvements in both elastin and collagen fibers of the skin as well as moisture content, likely via increased skin hyaluronic acid content.

Table 1. Average ( \pm standard deviation) of measured parameters at the start (T0) of the efficacy trial as well as 15 days (T1) and 30 day (T2) after daily application of face serum.

\begin{tabular}{lccc}
\hline Parameter & T0 & T1 & T2 \\
\hline Moisture (CU) & $41.853 \pm 12.391$ & $45.740 \pm 11.356^{*}$ & $50.520 \pm 11.823^{*}$
\end{tabular}




\begin{tabular}{lccc} 
Biological elasticity (R2) & $0.556 \pm 0.060$ & $0.609 \pm 0.062^{*}$ & $0.641 \pm 0.054^{*}$ \\
Relative elastic recovery (R7) & $0.382 \pm 0.055$ & $0.414 \pm 0.067^{*}$ & $0.430 \pm 0.047^{*}$ \\
Average wrinkle roughness, Ra (mm) & $0.044 \pm 0.012$ & $0.042 \pm 0.012^{*}$ & $0.038 \pm 0.012^{*}$ \\
Average wrinkle height, Rz (mm) & $0.163 \pm 0.046$ & $0.155 \pm 0.045^{*}$ & $0.143 \pm 0.043^{*}$ \\
Specular brightness rate & $0.322 \pm 0.220$ & $0.365 \pm 0.211^{*}$ & $0.403 \pm 0.216^{*}$ \\
\hline
\end{tabular}

Skin wrinkle width and depth parameters (Ra and $\mathrm{Rz}$, both measured in $\mathrm{mm}$ ) decreased by an average of $5.5 \%$ and $4.9 \%$ after 2 weeks $(p<0.01)$ and by $12.5 \%$ and $12.2 \%$ after 1 month ( $\mathrm{p}<0.01)$, respectively. All participants, except one, experienced a decrease of in both Ra and Rz skin roughness values after 30 days of treatment. An example of the decrease in lateral canthal lines in given in Figure 3. The subject in these images experienced an $11.5 \%$ decline in $\mathrm{Rz}$ values over the course of the trial as well as an $8.10 \%$ decrease in Ra values. The greater decrease in $\mathrm{Rz}$ values is readily discernable with the reduction in the amount of violet color, which corresponds to the greatest wrinkle depth, in the images captured during the latter two measurement dates. The participant featured in the images in Figure 5 had experience somewhat modest results when compared to 14 other volunteers who experience greater reductions in $\mathrm{Rz}$ values. Larger declines in $\mathrm{Ra}$ values that of the person in Figure 5 were also observed in twenty participants. The most substantial declines in $\mathrm{Rz}$ and Ra values were both $29.10 \%$.
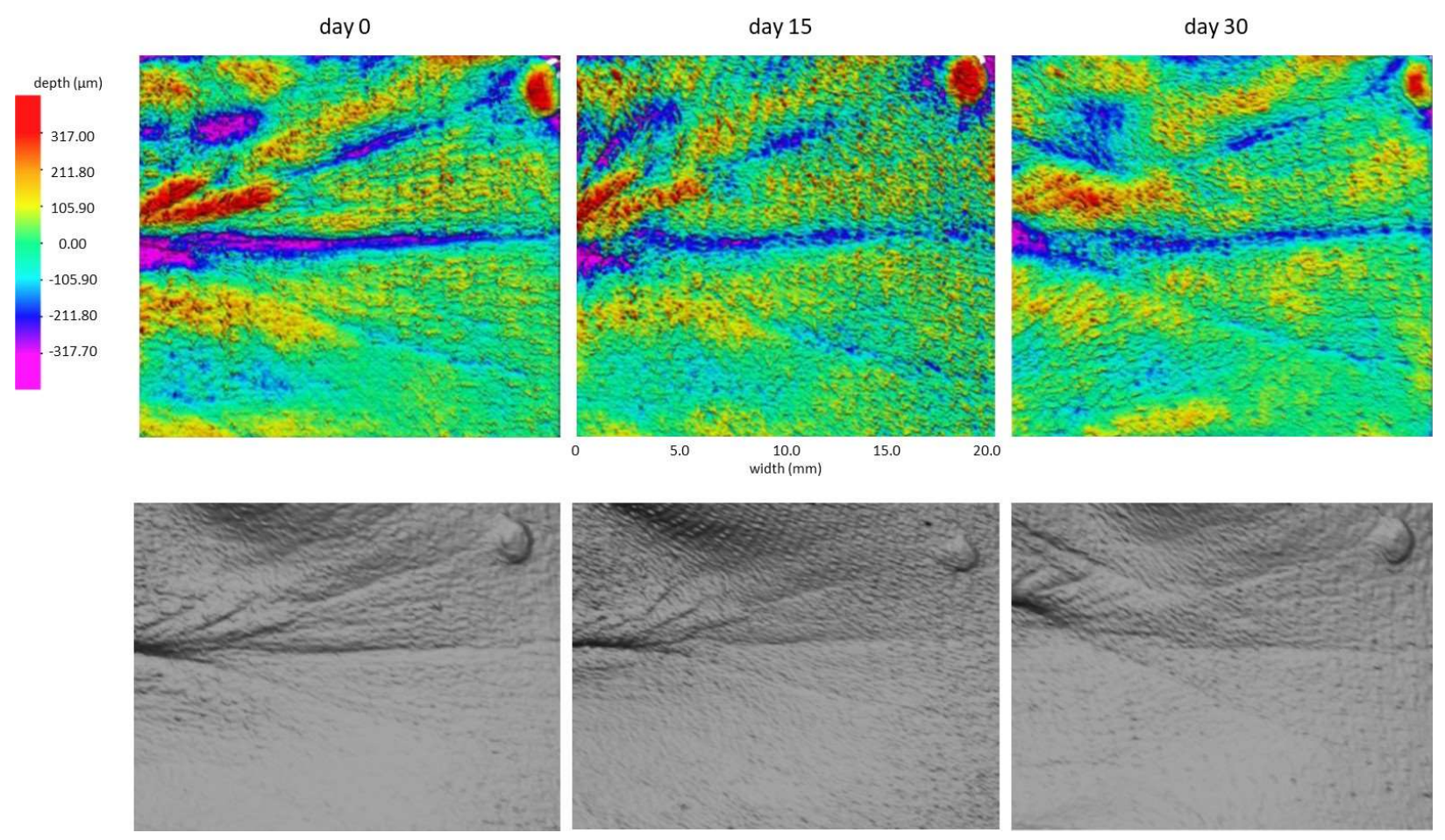

Figure 3. Example case of change in skin roughness (wrinkle depth and width) of lateral canthal lines, as visualized with DermaTOP Blue analysis. Corresponding black and white photos of the same facial area are given immediately below the depth and width colorscaled photos. 
The average brightness rate values rose from $0.322 \pm 0.220$ at $\mathrm{T} 0$ to $0.403 \pm 0.216$ at $\mathrm{T} 2$ $(\mathrm{p}<0.01)$. But the average individual percent change in specular brightness rate, from T0 to $\mathrm{T} 1$, was $22.5 \%$. By the end of the trial, this increased to $37.8 \%$. As with other parameters measured in this trial, all participants experienced an increase in specular brightness of the skin. The most dramatic change occurred in a Caucasian female whose values nearly doubled from 0.113 to 0.224 , a $98.23 \%$ increase. However, there was no evidence of any ancestry/ethnicity-dependent differential in skin brightness improvement, as there were overlapping minimum-maximum ranges for each skin type-4.4 to $25.3 \%$ for those of both African and European ancestry, 0.9 to $51.3 \%$ for those of Asian ancestry and 2.7 to $98.23 \%$ for those of European only ancestry. It can be reasonably expected that if a larger number of participants of Asian and African ancestry were included in the trial, even greater responses would be observed. It should also be noted that more than two-thirds of the trial participants experience greater than $10 \%$ increase in skin brightness, with a little over one-third having increases of $50 \%$ or more.

Dermatological evaluation of the facial skin of the trial participants revealed that the face serum did not increase the occurrence of seborrhea or increase the number of large pores. While not statistically significant, there was a trend of decreased comedones after one month of face serum application, especially on the cheek and forehead (Table 2).

Table 2. Number of comedones at start of trial (T0) and after 30 days of face serum application (T2).

\begin{tabular}{lcccc} 
& \multicolumn{2}{c}{ Total number comedones among participants } & \multicolumn{2}{c}{ Number of comedones/person (average \pm S.D.) } \\
& T0 & T2 & T0 & $1.13 \pm 0.43$ \\
Forehead & 53 & 34 & $1.77 \pm 0.73$ & $1.03 \pm 0.18$ \\
Cheekbone & 33 & 31 & $1.10 \pm 0.31$ & $1.33 \pm 0.48$ \\
Nose & 57 & 40 & $1.90 \pm 0.48$ & $1.03 \pm 0.18$ \\
Cheeks & 41 & 31 & $1.37 \pm 0.56$ & $1.27 \pm 0.52$ \\
Chin & 55 & 38 & $1.83 \pm 0.53$ & \\
\hline
\end{tabular}

In the self-assessment (Table 3), all participants reported experiencing improved in skin hydration. For the question regarding whether participants felt their skin was hydrated, the number reporting "not at all" or "a little" decreased from a combined $41 \%$ to $0 \%$ by the end of the trial, while the percent reporting "a lot" increased from $0 \%$ to $15 \%$ and those reporting "enough" increased by $26 \%$. This perception is consistent with the instrumental measurements. Most participants also reported experiencing significant increases in skin brightness, softness, radiance, elasticity, tone, and smoothness. All participants reported an overall improvement in facial skin, reduction in wrinkles and improvement in skin vitality after 1 month of face serum use. Among all self-assessment questions, no participants responded with "a lot" at T0. However, a substantial number of participants responded with "a lot" to the self-assessment questions by the end of the trial. Most volunteers noticed significant improvements in the crow's feet area. As with improved in skin hydration, the above participant perceptions are consistent with instrumental analyses and subsequent statistical analyses. This indicates the measured changes in skin quality are substantial enough for users of the face serum to feel and recognize them.

Table 3. Response percentage to self-assessment questions asked at the start of the efficacy trial (T0) and after 30 days of face serum application (T2).

\begin{tabular}{lccccc}
\hline & & \multicolumn{4}{c}{ Percentage of total responses } \\
& & A lot & Enough & A little & Not at all \\
\hline \multirow{2}{*}{ How hydrated does your skin feel? } & T0 & 0 & 59 & 37 & 4 \\
How bright is your skin? & T2 & 15 & 85 & 0 & 0 \\
\cline { 2 - 6 }
\end{tabular}


How soft is your skin?

How radiant is your skin?

How firm is your skin?

How elastic is your skin?

How smooth is your skin?

How tone is your skin?

\begin{tabular}{ccccc} 
T2 & 7 & 81 & 11 & 0 \\
\hline T0 & 11 & 63 & 22 & 4 \\
T2 & 30 & 67 & 4 & 0 \\
\hline T0 & 0 & 37 & 41 & 22 \\
T2 & 26 & 59 & 15 & 0 \\
\hline
\end{tabular}

$\begin{array}{lllll}\text { T0 } & 0 & 63 & 26 & 11\end{array}$

\begin{tabular}{ccccc} 
T2 & 26 & 52 & 22 & 0 \\
\hline T0 & 0 & 59 & 30 & 11
\end{tabular}

\begin{tabular}{ccccc} 
T2 & 30 & 59 & 11 & 0 \\
\hline T0 & 0 & 52 & 44 & 4
\end{tabular}

\begin{tabular}{ccccc} 
T2 & 30 & 56 & 11 & 4 \\
\hline T0 & 0 & 44 & 48 & 7 \\
T2 & 15 & 74 & 7 & 4
\end{tabular}

\section{Questions at conclusion of trial only}

How much has your facial skin improved overall?

How much more vital does your skin look?

Has the appearance of wrinkles and/or fine lines improved?

How satisfied are you with the efficacy of the face serum?

\begin{tabular}{llll}
19 & 67 & 15 & 0 \\
26 & 70 & 4 & 0 \\
19 & 59 & 22 & 0 \\
40 & 56 & 4 & 0 \\
\hline
\end{tabular}

The collective results of the repeat insult patch test and the efficacy clinical trial demonstrate that daily use of the face serum can provide benefits to skin health. In the efficacy trial, improvements occurred in each participant. This was especially true for moisture content and specular brightness. Skin hydration influences interkeratinocyte reflectance as well as other skin quality factors [27]. So, it naturally follows that improved hydration of the stratum corneum and underlying tissue will result in the outcomes observed in this study. The in vitro tyrosinase assay results demonstrated that the face serum had inhibitory activity, even at low concentrations. However, the activity was only modest. Therefore, it appears likely that the improvements in skin brightness are more dependent on hydrating properties of the face serum than on anti-tyrosinase potential.

In addition to the hydrating properties of the face serum, its ability to protect the extracellular matrix is evident in the results of the in vitro assay and clinical trial. The notable anti-collagenase activity will, of course, greatly impact the skin elasticity. Collagen degradation in the skin's extracellular matrix is a major aspect of the normal aging process. The resulting structural alterations reduce skin strength and resiliency, resulting in more prominent wrinkle formation [28]. Even so, the in vitro DPPH scavenging activity of the face serum points to significant antioxidant potential which may be the major factor in the positive outcomes observed in the efficacy trial.

With photoaging, collagen degradation and the associated adverse effects on skin quality are accelerated even further $[29,30]$. Photoaging begins with UV-induced generation of reactive oxygen species (ROS), where skin cell chromophores are excited in the presence of molecular oxygen. This produces ROS such as superoxide anion radicals (SAR), hydroxyl radicals and hydrogen peroxide [31]. When excessive UV exposure overwhelms endogenous antioxidant systems, ROS initiate a cascade of cellular signals leading to the transcription of collagenases (matrix metalloproteinases, or MMPs) in 
fibroblasts and an influx of elastase producing neutrophils [32, 33]. Increased MMP and elastase activity is responsible for extracellular matrix changes that result in the appearance of wrinkling, sagging, and other structural changes in the skin. Therefore, the reduction in skin roughness and improvements in skin elasticity are not only the results of collagenase inhibition but are likely the result of potent antioxidant activity of the ingredients in the face serum, such as bakuchiol and Kakadu plum extract. Bakuchiol is an excellent scavenger of linoleic acid peroxyl radicals, the DPPH radical, hydroxyl radicals, and glutathiyl radicals [34]. The antioxidant activity of bakuchiol is also known to prevent elevated expression of metalloproteinase-9 induction in vivo [35]. Further, Kakadu plum inhibited the induction of matrix metalloproteinase- 1 gene expression and subsequent collagen degradation in the skin UV-B exposed mice [36].

Aside from collagenase inhibition and antioxidant action, which prevent degradation of the extracellular matrix, some ingredients in the face serum promote the synthesis of new extracellular components. As discussed previously, palmitoyl tripetide-38 application increases the production of dermal and epidermal collagen, fibronectin and hyaluronic acid. Formulations containing this tripeptide have been previously evaluated in clinical trials. However, their efficacies have varied due to differences in ingredient composition and the face serum evaluated in the current study has a greater demonstrable impact on skin health. For example, the average $\mathrm{Rz}$ value declined by $9 \%$, with improvements recorded for $70 \%$ of volunteers, in a 28 -day trial of a palmitoyl tirpetptide-38, vitamin $C$ and vitamin E blend involving 35 women [37]. In our study, the face serum decreased average $\mathrm{Rz}$ values by $12.2 \%$, with recorded improvements for 29 out of 30 participants (97\%). Another trial evaluated the effect of daily application of a palmitoyl tripeptide-38, apple stem cell extract, creatine and urea blend on 32 women. This specific blend had a measurable anti-wrinkle effect in $71 \%$ of the women. The average increase in skin hydration (by Corneometer) was 13\%, with an average increase in skin elasticity (by Cutometer) was $10 \%$ after 28 days [38]. In our trial, the face serum improved wrinkles in $100 \%$ of the volunteers, improved average skin moisture by $23.82 \%$ and increase R2 and R7 elasticity measurements by 15 and 13\%, respectively. In a third clinical trial, 37 females evaluated the efficacy of a blend of palmitoyl tripeptide-38, rosemary leaf extract, tetrahexyldecylascorbate, Astragalus root extract and CoQ10 by self-assessment questionnaire after 12 weeks of daily topical application to the face [39]. After 4 weeks of use, only $21 \%$ of the subjects reported an improvement in the appearance of wrinkles. After 12 weeks, 83\% reported improved skin hydration. But in our study, $100 \%$ of the subjects reported improvements in both wrinkles and hydration within just one month. The ability of several compounds in the face serum to increase the hyaluronic acid content of the skin certainly limits transepidermal water loss, thereby enhancing moisture. The improved response observed in our study suggests that the combination of palmitoyl tripeptide-38, bakuchiol, Kakadu plum extract, allantoin, etc. in our face serum is more effective in improving skin hydration and the extracellular matrix.

\section{Conclusions}

The face serum is gentle to the skin. The combination of biologically active compounds in the serum possesses collagenase inhibiting properties and notable antioxidant activity in vitro. Clinical trial results also reveal that this combination may have a greater impact on skin health than topical treatments which may contain some of the same ingredients. It seems that the skin hydrating properties of the face serum are responsible, to a major degree, for the improvements in skin brightness and viscoelasticity. Additionally, protection of the extracellular matrix by inhibition of ROS-induced damage and increased expression of genes controlling dermal collagen, elastin and hyaluronic acid synthesis appear to be likely contributors to the improvements in skin quality. The qualitative assessments, or perception, of the participants match the instrumental analyses and demonstrate that the face serum's impact on skin health is clinically relevant. Therefore, daily topical application of the combination of active compounds in the face serum is a 
convenient and effective means of protecting facial skin and reducing the all too common signs of aging.

Author Contributions: Conceptualization, B.W., I.A. and S.D.; methodology, B.W., I.A. and S.D.; writing - original draft preparation, B.W.; writing-review and editing, I.A., S.D. All authors have read and agreed to the published version of the manuscript.

Funding: This research received no external organization funding.

Institutional Review Board Statement: The human studies were conducted according to the guidelines of the Declaration of Helsinki and approved by the ethic review boards of Mérieux NutriSciences (Acceptance No. 20.538598.0001) and Essex Testing Clinic (ETC No. 19549).

Informed Consent Statement: Informed consent was obtained from all subjects involved in the study.

Acknowledgments: The repeat insult patch test was completed with the assistance of John A. Erianne, Toni F. Miller and Annemarie E. Hollenback of Essex Testing Clinic, Inc. (Verona, New Jersey, USA). The efficacy trial was completed with the assistance of Massimo Gola and Emiliano Castellano of Merieux NutriSciences Corporation (Prato, Italy). Lucim Totale Face Serum was provided by Ariix LLC.

Conflicts of Interest: The authors are employed in the research and development department of New Age, Inc. a manufacturer of topical skin care products. The authors declare no other conflicts of interest.

\section{References}

1. Zouboulis, C.C. Human skin: an independent peripheral endocrine organ. Horm. Res. 2009; 54, 230-242. DOI: $10.1159 / 000053265$

2. Morrison, I.; Loken, L.S.; Olausson, H. The skin as a social organ. Exp. Brain Res. 2010; 204, 305-314. DOI: 10.1007/s00221-0092007-y.

3. Jafferany, M. Psychodermatology: a guide to understanding common psychocutaneous disorders. Prim. Care Companion J. Clin. Psychiatry 2007; 9, 203-213. DOI: 10.4088/pcc.v09n0306.

4. Tuckman, A. The potential psychological impact of skin conditions. Dermatol. Ther. (Heidelb.) 2017; 7 (Suppl 1), s53-s57. DOI: 10.1007/s13555-016-0169-7.

5. Nichols, T. Health utility, social interactivity, and peristomal skin status: a cross sectional study. J. Wound Ostomy Continence Nurs. 2018; 45, 438-443. DOI: 10.1097/WON.0000000000000457.

6. Honigman, R.; Castle, D.J. Aging and cosmetic enhancement. Clin. Interv. Aging 2006; 1, 115-119. DOI: 10.2147/ciia.2006.1.2.115.

7. Xin, Z.; Wu, X.; Ji, T.; Xu, B.; Han, Y.; Sun, M.; Jiang, S.; Li, T.; Hu, W.; Deng, C.; Yang, Y. Bakuchiol: a newly discovered warrior against organ damage. Pharmacol. Res. 2019; 141, 208-213. DOI: 10.1016/j.phrs.2019.01.001.

8. Chaudhuri, R.K.; Marchio, F. Bakuchiol in the management of acne-affected skin. Cosmet. Toil. 2011; 126, 502-510.

9. Mohanty, S.; Cock, I.E. The chemotherapeutic potential of Terminalia ferdinandiana: phytochemistry and bioactivity. Pharmacogn. Rev. 2012; 6, 29-36. DOI: 10.4103/0973-7847.95855.

10. Al-Niaimi, F.; Chiang, N.Y.Z. Topical vitamin C and the skin: mechanisms of action and clinical application. J. Clin. Aesthet. Dermatol. 2017, 10, 14-17.

11. Ugwu-Dike, P.; Nambudiri, V.E. A review of the ethnomedicinal uses of shea butter for dermatoses in Sub-Saharan Africa. Dermatol. Ther. 2021; e14786. DOI: 10.1111/dth.14786.

12. Andersson, A-C.; Alander, J. Shea butter extract for bioactive skin care. Cosmet. Toil. 2015; 130, 18-25.

13. Feily, A.; Namazi, M.R. Aloe vera in dermatology: a brief review. G. Ital. Dermatol. Venerol. 2009; 144, 85-91.

14. Araujo, L. U.; Grabe-Guimaraes, A.; Mosqueira, V.C.F.; Carneiro, C.M.; Silva-Barcellos, N.M. Profile of wound healing process induced by allantoin. Acta Cir. Bras. 2010, 25, 460-466. DOI: 10.1590/s0102-86502010000500014.

15. Spiers, S. M.; Cleaves, F.T. Topical treatment of the skin with a grapeseed oil composition. US Patent 5,916,573, filed 19 August 1999, and issued 29 June 1999.

16. Schagen, S.K. Topical peptide treatments with effective anti-aging results. Cosmetics 2017; 4, 16. DOI: 10.3390/cosmetics4020016.

17. Ferreira, S.M.; Magalhaes, M.C.; Sousa-Lobo, J.M.; Almeida, I.F. Trending anti-aging peptides. Cosmetics 2020; 7, 91. DOI: 10.3390/cosmetics7040091.

18. Foco, A.; Gasperlin, M.; Kristl, J. Investigation of liposomes as carriers of sodium ascorbyl phosphate for cutaneous photoprotection. Int. J. Pharm. 2005; 291, 21-29. doi: 10.1016/j.ijpharm.2004.07.039

19. Papakonstantinou, E.; Roth, M.; Karakiulakis, G.; Hyaluronic acid: a key molecule in skin aging. Dermatoendocrinol. 2012; 4, 253258. DOI: 10.4161/derm.21923.

20. Bukhari, S.N.A.; Roswandi, N.L.; Waqas, M.; Habib, H.; Hussain, F.; Khan, S.; Sohail, M.; Ramli, N.A.; Thu, H.E.; Hussain, Z. Hyaluronic acid, a promising skin rejuvenating biomedicine: a review of recent updates and pre-clinical and clinical 
investigations on cosmetic and nutricosmetic effects. Int. J. Biol. Macromol. 2018; 120 (Pt. B), 1682-1695. DOI: 10.1016/j.ijbiomac.2018.09.188.

21. Rietschel, R.L.; Fowler, J.F. Fisher's Contact Dermatitis, 4th ed., Williams \& Wilkins: Baltimore, Maryland, USA, 1995, pp. 836.

22. Matsuda, H.; Higashino, M.; Nakai, Y.; Inuma, M.; Kubo, M.; Lang, F.A. Studies of cuticle drugs from natural sources. IV. Inhibitory effects of some Arctostaphylos plants on melanin biosynthesis. Biol. Pharm. Bull. 1996; 19, 153-156. DOI: 10.1248/bpb.19.153.

23. West, B.J.; Deng, S.; Palu, A.K. Antioxidant and toxicity tests of roasted noni (Morinda citrifolia) leaf infusion. Int. J. Food Sci. Technol. 2009; 44, 2142-2146. DOI: 10.1111/j.1365-2621.2009.02051.x.

24. Everett, J.S.; Sommers, M.S. Skin viscoelasticity: physiologic mechanisms, measurement issues, and application to nursing science. Biol. Res. Nurs. 2013; 15, 338-346. doi: 10.1177/1099800411434151.

25. de Farias Pires T.; Azambuja, A.P.; Horimoto, A.R.; Nakamura, M.S.; de Oliveira Alvim, R.; Krieger, J.E.; Pereira, A.C. A population-based study of the stratum corneum moisture. Clin. Cosmet. Investig. Dermatol. 2016; 9, 79-87. doi:10.2147/CCID.S88485.

26. Paes, E.C.; Teepen, H.J.L.J.M.; Koop, W.A.; Kon, M. Perioral wrinkles: histologic differences between men and women. Aesthet. Surg. J. 2009; 29, 467-472. doi:10.1016/j.asj.2009.08.018.

27. Mandfredini, M.; Mazzaglia, G.; Ciardo, S.; Simonazzi, S.; Farnetani, F.; Longo, C.; Pellacani, G. Does skin hydration influence keratinocyte biology? In vivo evaluation of microscopic skin changes induced by moisturizers by means of reflectance confocal microscopy. Skin Res. Technol. 2013; 19, 299-307. DOI: 10.1111/srt.12042.

28. Rittie, L.; Fisher, G.J. Natural and sun-induced aging of human skin. Cold Spring Harb. Perspect. Med. 2015; 5, a015370. doi:10.1101/cshperspect.a015370.

29. Warren, R.; Gartstein, V.; Kligman, A.M.; Montagna, W.; Allendorf, R.A.; Ridder, G.M. Age, sunlight, and facial skin: a histologic and quantitative study. J. Am. Acad. Dermatol. 1981, 25, 751-670.

30. El-Domyati, M.; Attia, S.; Saleh, F.; Brown, D.; Birk, D.E.; Gasparro, F.; Ahmad, H.; Uitto, J. Intrinsic aging vs. photoaging: a comparative histopathological, immunohistochemical, and ultrastructural study of skin. Exp. Dermatol. 2002, 11, 398-405. doi:10.1034/j.1600-0625.2002.110502.x.

31. Hanson, K.M.; Simon, J.D. Epidermal trans-urocanic acid and the UV-A-induced photoaging of the skin. Proc. Natl. Acad. Sci. U.S.A. 1998; 95, 10576-10578. doi:10.1073/pnas.95.18.10576.

32. Brenneisen, P.; Wenk, J.; Klotz, L.O.; Wlaschek, M.; Brivina, K.; Krieg, T.; Scharffetter-Kochanek, K.; Central role of ferrous/ferric iron in the ultraviolet B irradiation-mediated signaling pathway leading to increased interstitial collagenase (matrix-degrading metalloprotease (MMP)-1) and stromelysin-1 (MMP-3) mRNA levels in cultured human dermal fibroblasts. J. Biol. Chem. 1998; 273, 5279-5287. Doi:10.1074/jbc.273.9.5279.

33. Rijken, F.; Bruijnzeel, P.L.B. The Pathogenesis of photoaging: the role of neutrophils and neutrophil-derived enzymes. J. Investig. Dermatol. Symp. Proc. 2009; 14, 67-72. doi:10.1038/jidsymp.2009.15.

34. Adhikari, S.; Joshi, R.; Patro, B.S.; Ghanty, T.K.; Chintalwar, G.J.; Sharma, A.; Chattopadhyay, S.; Mukherjee, T. Antioxidant activity of bakuchiol: experimental evidences and theoretical treatments on the possible involvement of the terpenoid chain. Chem. Res. Toxicol. 2003; 16, 1062-1069. DOI: 10.1021/tx034082r.

35. Liu, H.; Guo, W.; Guo, H.; Zhao, L.; Yue, L.; Li, X.; Feng, D.; Luo, J.; Wu, X.; Cui, W.; Qu, Y. Bakuchiol attenuates oxidative stress and neuron damage by regulating Trx1/TXNIP and the phosphorylation of AMPK after subarachnoid hemorrhage in mice. Front. Pharmacol. 2020; 11, 712. DOI: 10.3389/fphar.2020.00712.

36. Emy, S.; Iswari, I.S.; Winarti, N.W. Terminalia ferdinandiana inhibited the increase of matrix metalloproteinase-I and prevent collagen decrease in mice skin exposed to UV-B. Neurologico Spinale Medico Chirurgico 2021; 4, 11-14. DOI: 10.36444/nsmc.v4i1.136.

37. Lintner, K.; Gerstein, F.; Solish, N. A serum containing vitamins C \& E and a matric-repair tripeptide reduces facial signs of aging as evidenced by Primos ${ }^{\circledR}$ analysis and frequently repeated auto-perception. J. Cosmet. Dermatol. 2020; 19, 3262-3269. DOI: 10.1111/jocd.13770.

38. Sanz, M.T.; Campos, C.; Milani, M.; Foyaca, M.; Lamy, A.; Kurdian, K.; Trullas, C. Biorevitalizing effect of a novel facial serum containing apple stem cell extract, pro-collagen lipopeptide, creatine, and urea on skin aging signs. J. Cosmet. Dermatol. 2016; 15, 24-30. DOI: 10.1111/jocd.12173.

39. Herndon, J.H.; Jiang, L.; Kononov, T.; Fox, T. An open label clinical trial of a multiple-ingredient anti-aging moisturizer designed to improve the appearance of facial skin. J. Drugs Dermatol. 2015; 14, 699-704. 